\title{
Hamiltonian guiding center equations in a toroidal system
}

\author{
S. S. Abdullaev and K. H. Finken \\ Institut für Plasmaphysik, Forschungszentrum Jülich GmbH, EURATOM Association, \\ Trilateral Euregio Cluster, D-52425 Jülich, Germany
}

(Received 14 May 2002; accepted 27 June 2002)

\begin{abstract}
A Hamiltonian method to study the guiding center motion of charged particles in a toroidal magnetic system has been developed. It uses a cylindrical coordinate system instead of a magnetic coordinate system on which many conventional standard methods are based. The six-dimensional (6D) Hamiltonian equations for the guiding center motion are derived by a canonical transformation of fast-oscillating variables to slowly varying ones which are guiding center coordinates. It is shown that one of these slowly varying variables, i.e., the action variable conjugated to the fast-oscillating gyrophase is an adiabatic invariant for the tokamak equilibrium magnetic field perturbed by the external time-dependent magnetic field. This allows to reduce the 6D Hamiltonian system to the 4D one. The method is valid for the study of the guiding center motion of particles in time-dependent magnetic and electric fields, especially, ergodic magnetic fields, where spatial and temporal scales of variation are much larger than the gyroradius and the gyroperiod. (C) 2002 American Institute of Physics. [DOI: 10.1063/1.1502672]
\end{abstract}

\section{INTRODUCTION}

Since the early 1960s the Hamiltonian approach to studying the charged particle motion in magnetic fields has been subject to many studies ${ }^{1-7}$ because of its significance in magnetic fusion research. The equations of guiding center motion in Hamiltonian form has several advantages in the study of particle motion in magnetic confinement devices. It allows one to use the methods of the Hamiltonian theory, like methods of canonical transformations, the Hamilton-Jacobi method, Liouville's invariants, perturbation theory and the symplectic maps to efficiently solve the problem. Several Hamiltonian formulations of the guiding center motion have been proposed since the early 1980s (see Refs. 8-14). Some of these methods developed in Refs. 8-12 use magnetic coordinates ${ }^{15}$ as canonical variables and therefore would require the existence of magnetic surfaces. The case destroyed magnetic surfaces are treated either by assuming a special form for the magnetic field that gives rise to chaotic field lines, or using perturbation methods (see, e.g., Refs. 11 and 12). According to the more general approach proposed in Ref. 13, later generalized in Ref. 14 to time-dependent fields, one should find a coordinate system in which one of the covariant components of the magnetic vector $\mathbf{B}$ and the vector potential A vanish, e.g., $A_{r}=B_{r}=0$. These limitations on the form of the magnetic field may not be satisfied in some interesting cases of particle motion in tokamaks in the presence of the external magnetic perturbations. As an example, one can mention the tokamak with an ergodic divertor designed to control the heat and particle transport at the plasma edge by an externally created magnetic field (Refs. 16 and 17). All components of such a magnetic field with chaotic field lines are nonzero, ${ }^{18}$ and it is difficult to find a coordinate system in which one of the components of the field would vanish.

On the other hand, all these approaches are based on the existence of an adiabatic invariant, a magnetic moment $\mu$ $=m v_{\perp}^{2} / 2 B$, where $B=|\mathbf{B}|$ and $v_{\perp}$ is the velocity component perpendicular to the magnetic field. However, for some cases, for instance, in spherical tokamaks with a weak but strongly spatially varying magnetic field or in the presence of an electric field with strong gradients, the magnetic moment $\mu$ may break down (see Refs. 19 and 20). Under such circumstances the above methods may not work well. Moreover, as was shown in Refs. 21 and 22 even when the magnetic moment $\mu$ is a good adiabatic invariant in general magnetic configurations it is not a single-valued function of position $\mathbf{r}$. This makes it difficult to use the magnetic moment as an invariant in the solutions of the gyrokinetic equations.

In this work we propose a new Hamiltonian method to study the particle motion in a strong tokamak magnetic field using a cylindrical coordinate system. In its present form it is assumed that the toroidal field $B_{\varphi}$ decays inversely proportional to the radial coordinate $R, B_{\varphi} \propto R^{-1}$. This allows one to derive simple Hamiltonian equations for the guiding center motion of particles. The idea is similar to the one proposed by Mynick in Refs. 3 and 4 two decades ago for the mirror and field-reversed configurations in which the particle gyroradius is comparable to the system size. We formulate the canonical Hamiltonian equations in a cylindrical coordinate system $(R, \Phi, Z)$ to describe the particle motion in a tokamak magnetic field which may include the internal or external time-dependent magnetic and electric field perturbations as well. One can expect that this approach may be useful when the adiabatic invariance is broken, or the gyroradius is comparable to the characteristic spatial scale of variation of magnetic and electric fields.

The idea is that the Hamiltonian of the three-dimensional (3D) gyrating motion of a particle in strong magnetic and electric fields may be presented as a Hamiltonian for 1D motion along the radial coordinate $R$ in the effective poten- 
tial well $U=U\left(R ; Z, P_{z}, \Phi, P_{\varphi}, t\right)$, which is a steep function of $R$ and slowly varying along the other coordinates $(Z, \Phi)$ and time $t$, also the momentum $\left(P_{z}, P_{\varphi}\right)$, i.e., $H=P_{R}^{2} / 2$ $+U$. For a strong magnetic field, the motion along the radial coordinate is highly oscillatory near the minimum of $U(R)$ with the amplitude $\rho_{R}$ and the frequency $\omega_{R}$ being of the order of the gyroradius $r_{c}=e v_{\perp} B / m c$ and the gyrofrequency $\omega_{c}=e B / m c$, respectively. Introducing action-angle variables $\left(I_{R}, \vartheta_{R}\right)$ to describe such a motion by means of the canonical transformations of variables $\left(R, Z, \Phi, P_{R}, P_{z}, P_{\varphi}\right)$ $\rightarrow\left(\vartheta_{R}, \bar{Z}, \bar{\Phi}, I_{R}, \bar{P}_{z}, \bar{P}_{\varphi}\right)$ given by the generating function $S\left(R, Z, \Phi, I_{R}, \bar{P}_{z}, \bar{P}_{\varphi}\right)$ of mixed variables, allows one to derive the Hamiltonian equations for the guiding center variables $\left(\vartheta_{R}, \bar{Z}, \Phi, I_{R}, \bar{P}_{z}, \bar{P}_{\varphi}\right)$. A further canonical change of variables allows us to eliminate the radial gyrophase $\vartheta_{R}$, and thus to obtain a 4D system of Hamiltonian equations.

In Sec. II we derive the 6D and 4D Hamiltonian guiding center equations using the procedure described above. Simplified forms of these equations are obtained in Sec. III. The guiding center equations are verified in Sec. IV by comparing them with the results of a direct numerical integration of the original Hamiltonian system. The discussion of the results obtained are presented in Sec. V.

\section{HAMILTONIAN GUIDING CENTER EQUATIONS IN CYLINDRICAL COORDINATE SYSTEM}

In this section we derive the Hamiltonian for the guiding center motion in a toroidal system with coordinates $(\hat{R}, \varphi, \hat{Z})$. The particle motion is determined in the sixdimensional phase space (q,p) by the Hamiltonian equations:

$$
\frac{d q_{i}}{d T}=\frac{\partial H}{\partial p_{i}}, \quad \frac{d p_{i}}{d T}=-\frac{\partial H}{\partial q_{i}}, \quad i=1,2,3,
$$

where $\left(q_{1}, q_{2}, q_{3}\right)=(\hat{R}, \varphi, \hat{Z}), \quad\left(p_{1}, p_{2}, p_{3}\right)=\left(\hat{P}_{R}, \hat{P}_{\varphi}, \hat{P}_{Z}\right)$. The Hamiltonian of the system (1) is

$$
\begin{aligned}
H\left(\hat{R}, \varphi, \hat{Z}, \hat{P}_{R}, \hat{P}_{\varphi}, \hat{P}_{Z}, \hat{t}\right) & =\frac{1}{2 m}\left[\left(\hat{P}_{R}-\frac{e}{c} A_{R}\right)^{2}+\frac{1}{\hat{R}^{2}}\left(\hat{P}_{\varphi}-\frac{e}{c} \hat{R} A_{\varphi}\right)^{2}\right. \\
& \left.+\left(\hat{P}_{Z}-\frac{e}{c} A_{Z}\right)^{2}\right]+e \Phi
\end{aligned}
$$

where $\mathbf{A}=\mathbf{A}(\hat{R}, \varphi, \hat{Z}, \hat{t})=\left(A_{R}, A_{\varphi}, A_{Z}\right)$ is the magnetic vector potential, and $\Phi=\Phi(\hat{R}, \varphi, \hat{Z}, \hat{t})$ is the electric field.

For the sake of simplicity one can always set the radial component of the vector potential $A_{R}$ to be zero, i.e., $A_{R}$ $=0$, because of a gauge invariant of the vector potential. We suppose that the $z$ component of the vector potential $A_{Z}$ which determines the toroidal field $B_{\varphi}=-\partial A_{Z} / \partial \hat{R}$ be in form $A_{Z}=-B_{0} R_{0} \ln \hat{R}$, i.e., $B_{\varphi}=B_{0} R_{0} / \hat{R}$, where $B_{0}$ is a strength of magnetic field at the major radius of torus $\hat{R}$ $=R_{0}$. We also assume that the equilibrium poloidal magnetic field of the plasma $\left(B_{R}, B_{Z}\right)$ and the perturbed magnetic field created by the external coils may completely be described by the toroidal component of the vector potential $A_{\varphi}(\hat{R}, \varphi, \hat{Z}, \hat{t}): B_{Z}=\hat{R}^{-1} \partial\left(\hat{R} A_{\varphi}\right) / \partial \hat{R}, B_{R}=-\partial A_{\varphi} / \partial \hat{Z}$.

In a realistic magnetic equilibrium the toroidal field $B_{\varphi}$ may deviate from $B_{\varphi}=B_{0} R_{0} / \hat{R}$ due to, for instance, a diamagnetic current in the plasma. The method presented below may be generalized to include this case as well.

Furthermore, we will use the the normalized coordinates $x=\left(\hat{R}-R_{0}\right) / R_{0}, z=\hat{Z} / R_{0}$, the normalized time $t=\omega_{c} \hat{t}$, and the normalized momenta:

$$
p_{x}=\frac{\hat{P}_{x}}{m \omega_{c} R_{0}}, \quad p_{\varphi}=\frac{\hat{P}_{\varphi}}{m \omega_{c} R_{0}^{2}}, \quad p_{z}=\frac{\hat{P}_{Z}-e A_{0 z} / c}{m \omega_{c} R_{0}},
$$

where $\omega_{c}=e B_{0} / m c$ is a reference gyrofrequency, $A_{0 z}$ $=A_{Z}\left(R_{0}\right)=\ln R_{0}$. The corresponding Hamiltonian function has the form

$$
\begin{aligned}
& \mathcal{H}\left(x, \varphi, z, p_{x}, p_{\varphi}, p_{z}, t\right) \\
& \quad=\frac{1}{2}\left[p_{x}^{2}+\frac{\left(p_{\varphi}-f\right)^{2}}{(1+x)^{2}}+\left(p_{z}+\ln (1+x)\right)^{2}\right]+\phi(x, \varphi, z, t),
\end{aligned}
$$

where $\mathcal{H}=H /\left(m \omega_{c}^{2} R_{0}^{2}\right)$ is the normalized Hamiltonian, $\phi(x, \varphi, z, t)=e \Phi(\hat{R}, \varphi, \hat{Z}, \hat{t}) /\left(m \omega_{c}^{2} R_{0}^{2}\right)$ is a normalized electric potential and $f \equiv f(x, z, \varphi, t)=R A_{\varphi}(\hat{R}, \varphi, \hat{Z}, \hat{t}) /\left(R_{0}^{2} B_{0}\right)$ is a normalized $\varphi$ component of the vector potential $A_{\varphi}$. We assume that the toroidal magnetic field $B_{\varphi}$ is larger than the poloidal magnetic field $\left(B_{R}, B_{Z}\right)$ and the magnetic perturbation field $\mathbf{B}_{\text {pert }}$.

The Hamiltonian (3) may be given as $\mathcal{H}=p_{x}^{2} / 2+U$, where

$$
\begin{aligned}
& U\left(x ; \varphi, z, p_{\varphi}, p_{z}, t\right) \\
& \quad=\frac{1}{2}\left[\frac{\left(p_{\varphi}-f\right)^{2}}{(1+x)^{2}}+\left(p_{z}+\ln (1+x)\right)^{2}\right]+\phi(x, \varphi, z, t),
\end{aligned}
$$

is the effective potential for the one-dimensional motion along the radial coordinate $x$. Since the poloidal magnetic field determined by $f$ is smaller than the toroidal field $B_{t}$, and the electric field $\phi$ is much smaller than the vector potential $A_{z}$, the radial dependence of the potential function $U$ mainly depends on the second term on the right hand-side of (4), i.e., on $U_{z}(x)=\left(p_{z}+\ln (1+x)\right)^{2}$. The effective potential $U$ has a minimum near $x=x_{c}$ where $U_{z}\left(x_{c}\right)=0$ and sharply grows with increasing distance $\left|x-x_{c}\right|$. The shape of the potential $U$ along the $x$ coordinate is shown in Fig. 1 for the values of particle energy $\left(\mathrm{H}^{+}\right)$and for the typical parameters of the Torus Experiment for the Technology-Oriented Research (TEXTOR): ${ }^{17} B_{0}=2.25 \mathrm{~T}, R_{0}=175 \mathrm{~cm}, a=46 \mathrm{~cm}$.

Particles trapped inside the potential well oscillate along the radial axis about their minimum $x_{c}$. The amplitude of oscillations depends on the particle energy. For an amplitude of oscillations much smaller than the characteristic spatial scale of variation of the magnetic field the potential $U$ may be expanded in a series of powers $\left(x-x_{c}\right)$ near its minimum at $x_{c}$,

$$
U=U_{0}+\frac{U_{0}^{\prime \prime}}{2}\left(x-x_{c}\right)^{2}+\sum_{k \geqslant 3} a_{k}\left(x-x_{c}\right)^{k},
$$




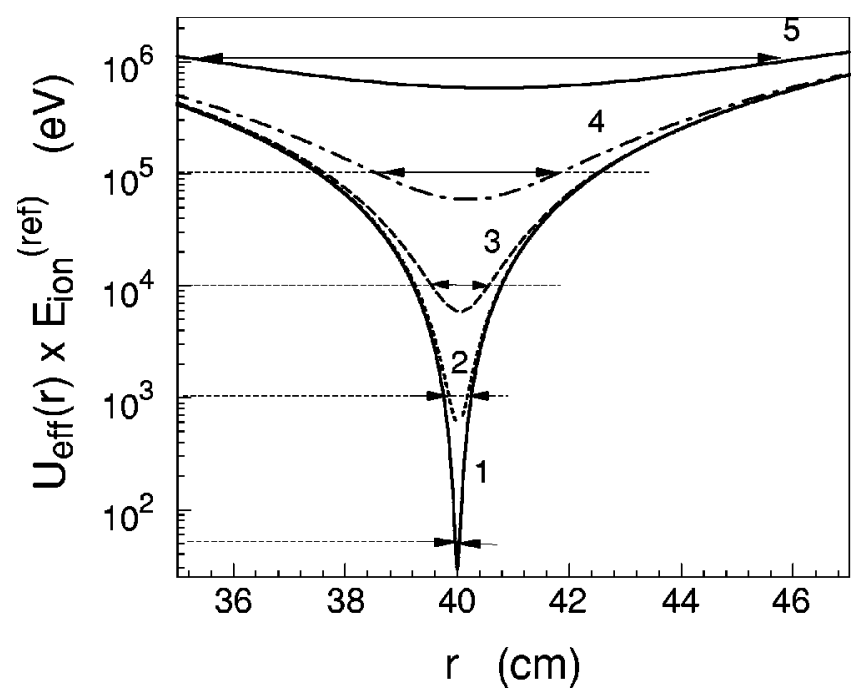

FIG. 1. Radial dependencies of the effective potential $U_{\text {eff }}(x)$ $=U\left(x ; z, \phi, p_{z}, p_{\varphi}, t\right)$ for different values of ion $\left(\mathrm{H}^{+}\right)$energy $E$. The tokamak magnetic field is described by the standard equilibrium magnetic field (see Sec. IV). Curve 1 corresponds to the energy $E=50 \mathrm{eV}, 2-E=1 \mathrm{keV}, 3$ $-E=10 \mathrm{keV}, 4-E=100 \mathrm{keV}$, and $5-E=1 \mathrm{MeV}$. The corresponding horizontal arrows show the radial extend of particle oscillations. Here $E_{\text {ion }}^{(\text {ref })}=m \omega_{c}^{2} R_{0}^{2}$ is a reference ion $\left(\mathrm{H}^{+}\right)$energy, $r=R_{0} x, R_{0}=175 \mathrm{~cm}$.

where $U_{0}=U_{0}\left(z, p_{z}, \varphi, p_{\varphi}, t\right) \equiv U\left(x_{c} ; z, \varphi, p_{z}, p_{\varphi}, t\right)$, and $a_{k}$ $=U_{0}^{(k)} / k$ ! are expansion coefficients. The minimum point $x_{c}$, i.e., $\partial U / \partial x=0$, is determined by the equation

$$
\begin{aligned}
\left.\frac{\partial U}{\partial x}\right|_{x=x_{c}}= & -\left[\frac{\left(p_{\varphi}-f\right)^{2}}{(1+x)^{3}}+\frac{\left(p_{\varphi}-f\right)}{(1+x)^{2}} \frac{\partial f}{\partial x}-\frac{p_{z}+\ln (1+x)}{1+x}\right. \\
& \left.+\frac{\partial \phi}{\partial x}\right]\left.\right|_{x=x_{c}}=0
\end{aligned}
$$

Then the Hamiltonian takes the form

$$
\begin{aligned}
\mathcal{H}\left(x, z, \varphi, p_{x}, p_{z} p_{\varphi}, t\right) & \\
= & \frac{1}{2}\left[p_{x}^{2}+\omega_{x}^{2}\left(x-x_{c}\right)^{2}\right]+\sum_{k \geqslant 3} a_{k}\left(x-x_{c}\right)^{k} \\
& +U_{0}\left(z, p_{z}, \varphi, p_{\varphi}, t\right) .
\end{aligned}
$$

The quantity $\omega_{x}=\sqrt{U_{0}^{\prime \prime}}$ is the frequency of radial oscillations

$$
\begin{aligned}
\omega_{x}= & \left.\left(\frac{\partial^{2} U}{\partial x^{2}}\right)^{1 / 2}\right|_{x=x_{c}} \\
= & \frac{1}{1+x_{c}}\left[1-u_{z}+3 u_{\varphi}^{2}+4 u_{\varphi} \frac{\partial f}{\partial x}+\left(\frac{\partial f}{\partial x}\right)^{2}\right. \\
& \left.-\left(1+x_{c}\right) u_{\varphi} \frac{\partial^{2} f}{\partial x^{2}}+\left(1+x_{c}\right)^{2} \frac{\partial^{2} \phi}{\partial x^{2}}\right]^{1 / 2},
\end{aligned}
$$

where $\quad u_{\phi}=v_{\phi} / \omega_{c} R_{0}=\left(p_{\varphi}-f_{0}\right) /\left(1+x_{c}\right), \quad u_{z}=v_{z} / \omega_{c} R_{0}$ $=p_{z}+\ln \left(1+x_{c}\right)$ are normalized velocities. For moderately energetic particles the frequency $\omega_{x}$ is of order of 1, i.e., the frequency of the radial oscillations $\omega_{R}=\omega_{x} \omega_{c}$ is in the same order as the gyrofrequency $\omega_{c}$.

The Hamiltonian (7) describes the fast oscillating motion along the $x$ coordinate around the center $x_{c}$ with the fre- quency $\omega_{x}$. Both, the center $x_{c}$ and the frequency $\omega_{x}$ are slowly varying functions of spacial coordinates $(z, \varphi)$, time $t$, and the canonical momenta $p_{z}, p_{\varphi}$, i.e., $x_{c}$ $=x_{c}\left(z, \varphi, p_{z}, p_{\varphi}, t\right)$ and $\omega_{x}=\omega_{x}\left(z, \varphi, p_{z}, p_{\varphi}, t\right)$. The equation for the guiding center $x_{c}$ may be rewritten

$$
u_{\varphi}^{2}+u_{\varphi} \frac{\partial f}{\partial x}-u_{z}-(1+x) \frac{\partial \phi}{\partial x}=0 .
$$

In the original variables this equation describes the balance of the centripetal force $F_{c}=m v_{\phi}^{2} / R$ and the radial component of the Lorentz force $F_{L R}=e E+(e / c)\left(v_{\phi} B_{Z}\right.$ $\left.-v_{Z} B_{\phi}\right)$ :

$$
\frac{m v_{\phi}^{2}}{R}=e E_{R}+\frac{e}{c}\left(v_{\varphi} B_{Z}-v_{Z} B_{\varphi}\right),
$$

where $E_{R}=-\partial \Phi / \partial R$.

Transformation to slowly varying variables. The direct integration of the system of Hamiltonian equations (1) requires very long computational times because the integration time steps should be small as compared to the gyro-period. In order to simplify the problem one can change the fast oscillating variables in Eq. (1) to slowly varying ones. The integration of the equations with respect to the latter variables would not be time consuming.

The Hamiltonian system (7) is similar to a onedimensional oscillator with a slowly varying frequency of oscillations $\omega_{x}$ and the equilibrium point $x_{c}$ in space and time. For small amplitude of the radial oscillations, where one can neglect the expansion terms higher than $k>2$, the problem is similar to the well-known problem of the harmonic oscillator under slow changes in the frequency, which has been studied by many authors for a long time (see, e.g., Refs. 23-25). If the gyration period $2 \pi / \omega_{x}$ is much smaller than the time scale $t_{B}$ of a change of $\omega_{x}$ and $x_{c}$ and the transition time of the system $L / v_{T}$, where $L$ is spatial scale of change of the magnetic field, and $v_{T}$ is a thermal velocity of a particle, then the action variable $I_{x}$ defined as $I_{x}$ $=\left[p_{x}^{2} / \omega_{x}+\omega_{x}\left(x-x_{c}\right)^{2}\right] / 2$ is the adiabatic invariant. The existence of the adiabatic invariant significantly simplifies the study of particle motion in an inhomogeneous magnetic field.

More rigorously, the adiabatic invariant $I_{x}$ can be introduced by the canonical transformation of variables from the variables $\left(x, z, \varphi, p_{x}, p_{z}, p_{\varphi}\right)$ to the new ones ( $\vartheta_{x}, Z, \Phi, I_{x}, P_{z}, P_{\varphi}$ ) determined by the generating function $F=F\left(x, z, \varphi, I_{x}, P_{z}, P_{\varphi}, t\right)$ of mixed variables, old coordinates $q_{i}=(x, z, \varphi)$ and new momenta $P_{i}=\left(I_{x}, P_{z}, P_{\varphi}\right), \quad(i$ $=1,2,3)$. Then the old momenta $p_{i}=(x, z, \varphi)$ and the new coordinates $Q_{i}=\left(\vartheta_{x}, Z, \Phi\right)$ are determined by $p_{i}=\partial F / \partial q_{i}$, $Q_{i}=\partial F / \partial P_{i}$. We will seek the generating function $F$ in the form

$$
\begin{aligned}
F\left(x, I_{x}, z, P_{z}, \varphi, P_{\varphi}, t\right)= & z P_{z}+\varphi P_{\varphi} \\
& +S\left(x, I_{x}, \epsilon z, \epsilon P_{z}, \epsilon \varphi, \epsilon P_{\varphi}, t\right),
\end{aligned}
$$

where $S$ is the part of the generating function which is slowly varying with respect to the variables $\left(z, \varphi, P_{z}, P_{\varphi}\right)$ as well as to the time $t$, 


$$
\begin{aligned}
\epsilon S\left(x, I_{x}, z, P_{z}, \varphi, P_{\varphi}, t\right) \\
\quad=\int^{x} p_{x}\left(x^{\prime} ; I_{x}, z, P_{z}, \varphi, P_{\varphi}, t\right) d x^{\prime},
\end{aligned}
$$

where

$$
\begin{aligned}
p_{x}\left(x ; I_{x}, z, P_{z}, \varphi, P_{\varphi}\right)= & {\left[2\left(\mathcal{H}-U_{0}\right)-\omega_{0}^{2}\left(x-x_{c}\right)^{2}\right.} \\
& \left.-\sum_{k \geqslant 3} a_{k}\left(x-x_{c}\right)^{k}\right]^{1 / 2} .
\end{aligned}
$$

Here $\epsilon$ is a small dimensionless parameter of the order of $\rho_{x} / L$ where $\rho_{x}$ is the amplitude of radial oscillations and $L$ is a characteristic spatial scale of the magnetic field variation. The generating function $F$ defines the "close to the identity" transformation of variables $\left(z, \varphi, p_{z}, p_{\varphi}\right)$ and transforms the fast variables $\left(x, p_{x}\right)$ into the action-angle variables $\left(\vartheta_{x}, I_{x}\right)$ :

$$
I_{x}=\frac{1}{2 \pi} \oint_{C} p_{x} d x, \quad \vartheta_{x}=\frac{\partial S}{\partial I_{x}},
$$

where the integral is taken along the closed curve $C$ of constant $\mathcal{H}=\mathcal{H}\left(x, z, \varphi, p_{x}, p_{z}, p_{\varphi}, t\right)$ on the $\left(x, p_{x}\right)$ plane. Here $U_{0}=U_{0}\left(z, \varphi, P_{z}, P_{\varphi}, t\right), \quad x_{c}=x_{c}\left(z, \varphi, P_{z}, P_{\varphi}, t\right)$, and $\omega_{0}$ $=\omega_{0}\left(z, \varphi, P_{z}, P_{\varphi}, t\right)=\omega_{x}\left(x_{c}, z, \varphi, P_{z}, P_{\varphi}, t\right)$ are slowly varying functions of the their arguments $z, \varphi, P_{z}, P_{\varphi}, t$.

For small radial gyro-oscillations one can neglect higher order expansion terms $k \geqslant 3$. Then for the generating function $S$ one can obtain

$$
S\left(x, I_{x}, z, P_{z}, \varphi, P_{\varphi}, t\right)=I_{x}\left(\arcsin y+y \sqrt{1-y^{2}}\right),
$$

where $y=\left(x-x_{c}\right) / \sqrt{2 I_{x} / \omega_{x}}$. Using (13) we have the following relations between old and new variables:

$$
\begin{aligned}
& \vartheta_{x}=\frac{\partial F}{\partial I_{x}}=\arcsin \frac{x-x_{c}}{\sqrt{2 I_{x} / \omega_{0}}}, \\
& p_{x}=\frac{\partial F}{\partial x}=\sqrt{2 I_{x} \omega_{0}} \cos \vartheta_{x}, \\
& p_{i}=\frac{\partial F}{\partial q_{i}}=P_{i}+\epsilon \frac{\partial S}{\partial q_{i}}, \\
& Q_{i}=\frac{\partial F}{\partial P_{i}}=q_{i}+\epsilon \frac{\partial S}{\partial P_{i}} \quad(i=2,3),
\end{aligned}
$$

where

$$
\begin{aligned}
\epsilon \frac{\partial S}{\partial \eta} & =\left(I_{x} \sin \vartheta_{x} \frac{\partial \ln \omega_{0}}{\partial \eta}-\sqrt{2 I_{x} \omega_{0}} \frac{\partial x_{c}}{\partial \eta}\right) \cos \vartheta_{x} \\
& =-\rho_{x} \omega_{0} \frac{\partial x_{c}}{\partial \eta} \cos \vartheta_{x}+\frac{\rho_{x}^{2}}{4} \frac{\partial \omega_{0}}{\partial \eta} \sin 2 \vartheta_{x} .
\end{aligned}
$$

Here $\eta=\left(z, \varphi, P_{z}, P_{\varphi}\right), \quad\left(q_{2}, q_{3}\right)=\left(I_{x}, p_{z}, p_{\varphi}\right), \quad\left(Q_{2}, Q_{3}\right)$ $=(Z, \Phi) \quad(i=1,2,3)$. From (14) follows that $x=x_{c}$ $+\rho_{x} \sin \vartheta_{x}$, where $\rho_{x}=\sqrt{2 I_{x} / \omega_{0}}$ is a gyroradius along the $x$ axis.

According to (7), (14), (15), the new Hamiltonian $H$ determined as $H=\mathcal{H}+\partial F / \partial t$ takes the form

$$
H\left(\vartheta_{x}, Z, \Phi, I_{x}, P_{z}, P_{\varphi}, t\right)=\widetilde{\omega}_{0} I_{x}+\widetilde{U}_{0}+\epsilon \frac{\partial S}{\partial \epsilon t},
$$

where

$$
\begin{aligned}
\widetilde{\omega}_{0}= & \omega_{0}\left(Z-\epsilon \frac{\partial S}{\partial \epsilon P_{z}}, \Phi-\epsilon \frac{\partial S}{\partial \epsilon P_{\varphi}}, P_{z}+\epsilon \frac{\partial S}{\partial \epsilon z}, P_{\varphi}\right. \\
& \left.+\epsilon \frac{\partial S}{\partial \epsilon \varphi}, t\right), \\
\widetilde{U}_{0}= & U_{0}\left(Z-\epsilon \frac{\partial S}{\partial \epsilon P_{z}}, \Phi-\epsilon \frac{\partial S}{\partial \epsilon P_{\varphi}}, P_{z}+\epsilon \frac{\partial S}{\partial \epsilon z}, P_{\varphi}\right. \\
& \left.+\epsilon \frac{\partial S}{\partial \epsilon \varphi}, t\right) .
\end{aligned}
$$

Unlike the original stiff Hamiltonian (7), the new Hamiltonian (17) describes the motion of particles in slowly varying variables $\left(Z, \Phi, I_{x}, P_{z}, P_{\varphi}\right)$, clearly extracting the highfrequency gyro-oscillations into the gyro-phase $\vartheta_{x}$ :

$$
\begin{array}{ll}
\frac{d \vartheta_{x}}{d t}=\frac{\partial H}{\partial I_{x}}, & \frac{d I_{x}}{d t}=-\frac{\partial H}{\partial \vartheta_{x}}, \\
\frac{d Z}{d t}=\frac{\partial H}{\partial P_{z}}, & \frac{d P_{z}}{d t}=-\frac{\partial H}{\partial Z}, \\
\frac{d \Phi}{d t}=\frac{\partial H}{\partial P_{\varphi}}, & \frac{d P_{\varphi}}{d t}=-\frac{\partial H}{\partial \Phi} .
\end{array}
$$

The coordinates $\left(x_{c}, Z, \Phi\right)$ describe the guiding center of the gyro motion of a particle. According to (14), (15), and (16) the particle coordinates $(x, z, \varphi)$ up to the first order of $\epsilon$ are related to these coordinates as

$$
\begin{aligned}
& x=x_{c}+\rho_{x} \sin \vartheta_{x}, \\
& z=Z-\frac{\partial S}{\partial P_{z}}=Z+\rho_{x} \omega_{0} \frac{\partial x_{c}}{\partial P_{z}} \cos \vartheta_{x}, \\
& \varphi=\Phi-\frac{\partial S}{\partial P_{\varphi}}=\Phi+\rho_{x} \omega_{0} \frac{\partial x_{c}}{\partial P_{\varphi}} \cos \vartheta_{x} .
\end{aligned}
$$

Using the fact that the action variable $I_{x}$, conjugated to the gyrophase $\vartheta_{x}$, is an adiabatic invariant one can further simplify the system (18)-(20). In the next section we will show that $I_{x}$ is conserved during the slow drift motion of the particle with sufficiently high accuracy. We will show furthermore that the action $I_{x}$ is very close the main adiabatic invariant, the magnetic moment of the particle $\mu$ $=m v_{\perp}^{2} / 2 B$, where $\mathbf{v}_{\perp}$ is the of the particle velocity perpendicular to the magnetic field $\mathbf{B}(B=|\mathbf{B}|)$. The existence of the adiabatic invariant $I_{x}$ allows us to reduce the 6D system (18) -(20) to a 4D system of Hamiltonian equations. For a small ratio $\epsilon=\rho_{x} / L \ll 1$ this procedure may be studied using perturbative methods with a successive canonical change of variables. $^{28}$

Using the smallness of the ratio $\epsilon$ one can expand the Hamiltonian (17) in powers of $\epsilon$. Neglecting the small terms of order of $\epsilon^{2}$ and higher, we can present the Hamiltonian in the form 


$$
\begin{aligned}
H\left(\vartheta_{x}, Z, \Phi, I_{x}, P_{z}, P_{\varphi}, t\right)= & H_{0}\left(Z, \Phi, I_{x}, P_{z}, P_{\varphi}, t\right) \\
& +\epsilon H_{1}\left(\vartheta_{x}, Z, \Phi, I_{x}, P_{z}, P_{\varphi}, t\right),
\end{aligned}
$$

where the first term $H_{0}$ on the right-hand side describes the part of the Hamiltonian which does not depend on the gyrophase $\vartheta_{x}$ :

$$
\begin{aligned}
H_{0}\left(Z, \Phi, I_{x}, P_{z}, P_{\varphi}, t\right)= & \omega_{0}\left(Z, \Phi, P_{z}, P_{\varphi}, t\right) I_{x} \\
& +U_{0}\left(Z, \Phi, P_{z}, P_{\varphi}, t\right) .
\end{aligned}
$$

The fast-oscillating second term $H_{1}$ in (22) up to the first order of $\epsilon$ is determined by

$$
\begin{aligned}
\epsilon H_{1} & \left(\vartheta_{x}, Z, \Phi, I_{x}, P_{z}, P_{\varphi}, t\right) \\
& =-\rho_{x} \omega_{0} \cos \vartheta_{x} F\left(I_{x}, Z, \Phi, P_{z}, P_{\varphi}, t\right),
\end{aligned}
$$

where

$$
\begin{aligned}
F\left(I_{x}, Z, \Phi, P_{z}, P_{\varphi}, t\right)= & \sum_{i=1,2}\left[\frac{\partial\left(\omega_{0} I_{x}+U_{0}\right)}{\partial P_{i}} \frac{\partial x_{c}}{\partial Q_{i}}\right. \\
& \left.-\frac{\partial\left(\omega_{0} I_{x}+U_{0}\right)}{\partial Q_{i}} \frac{\partial x_{c}}{\partial P_{i}}\right]+\frac{\partial x_{c}}{\partial t} .
\end{aligned}
$$

The unperturbed Hamiltonian $H_{0}$ does not depend on the gyrofrequency, and hence the action variable $I_{x}$ is an integral of motion. In an axisymmetric and time-independent tokamak magnetic configuration, i.e., $\partial H_{0} / \partial \Phi \equiv 0, \partial H_{0} / \partial t \equiv 0$ there exist two more integrals of motion $I_{z}$ and $I_{\varphi} \equiv P_{\varphi}$ (the action variables) related to the slow drift motion in poloidal and toroidal coordinates, particularly, the action $I_{z}$ is defined by $I_{z}=(1 / 2 \pi) \oint_{C} P_{z} d Z$, where the integral is taken along the closed curve $C$, the projection of the orbit into the $\left(Z, P_{z}\right)$ plane. Then the unperturbed system $H_{0}=H_{0}\left(I_{x}, I_{z}, I_{\varphi}\right)$ (23) is completely integrable. The frequency $\omega_{x}=\partial H_{0} / \partial I_{x}$ determines the fast radial gyro-oscillations, while $\omega_{z}=\partial H_{0} / \partial I_{z}$ and $\omega_{\varphi}=\partial H_{0} / \partial I_{\varphi}$ are slow frequencies of conditionally periodic drift motion along vertical $z$ and toroidal $\varphi$ coordinates on the drift toroidal surfaces.

Such a conditionally periodic motion of particles may also survive if the nonaxisymmetric (or time-dependent) magnetic perturbation is present. However, in this case several groups of resonant drift surfaces (with $m \omega_{z}-n \omega_{\varphi}$ $+l \Omega=0$ ) are destroyed and the integrals $I_{z}$ and $I_{\varphi}$ may not exist. If the interaction of these resonant drift surfaces is sufficiently strong, it gives rise to chaotic drift motion of particles, similar to the ones of resonant magnetic surfaces.

The influence of the small perturbation term (24) containing the gyrophase $\vartheta_{x}$ on the described above drift motion of Hamiltonian system (23) may be studied using the classical perturbation methods of the KAM (KolmogorovArnold-Moser) theory. First of all the perturbation term (24) of the first order $\epsilon$ may be eliminated by the canonical transformation of variables $\left(\vartheta_{x}, Z, \Phi, I_{x}, P_{z}, P_{\varphi}\right)$ $\rightarrow\left(\bar{\vartheta}_{x}, \bar{Z}, \bar{\Phi}, J_{x}, \bar{P}_{z}, \bar{P}_{\varphi}\right)$ transforming the Hamiltonian (22) into

$$
\begin{aligned}
\bar{H}= & \bar{H}_{0}\left(\bar{Z}, \Phi, J_{x}, \bar{P}_{z}, \bar{P}_{\varphi}, t ; \epsilon\right) \\
& +\epsilon^{2} H_{2}\left(\bar{\vartheta}_{x}, \bar{Z}, \bar{\Phi}, J_{x}, \bar{P}_{z}, \bar{P}_{\varphi}\right),
\end{aligned}
$$

with $J_{x}=I_{x}-\epsilon \partial S_{1} / \partial \vartheta_{x}$. The new Hamiltonian (25) contains the gyrophase $\vartheta_{x}$ in the terms of order of $\epsilon^{2}$. Further successive canonical transformations of variables shift the terms with the gyrophase to the order $\epsilon^{4}, \epsilon^{8}$ and so on. Then the transformed action variable $J_{x}$ will be an integral of motion with sufficiently high accuracy in $\epsilon$. The existence of such successive canonical transformations is an immediate consequence of the KAM theory ${ }^{28}$ in the case where the unperturbed Hamiltonian $H_{0}(23)$ is integrable. In the latter case the fast frequency $\omega_{x}$ of radial gyro-oscillations is much larger than both slow drift frequencies $\omega_{z}$ and $\omega_{\varphi}$, i.e., the resonance condition $\omega_{x}-m \omega_{z}-n \omega_{\varphi}=0$ is never satisfied even for moderately large mode numbers $(m, n)$. In the nonintegrable case of drift motion, i.e., in a case of chaotic drift motion, the existence of such canonical transformations is not obvious from the KAM theory. However, one can intuitively expect that a slow chaotic drift motion does not change the above result.

The elimination of the gyrophase $\vartheta_{x}$ allows one to reduce the 6D system of the guiding center equations (18)(20) to the four-dimensional system for its guiding center variables $\left(Z, \Phi, P_{z}, P_{\varphi}\right)$ :

$$
\begin{array}{ll}
\frac{d Z}{d t}=\frac{\partial H}{\partial P_{z}}, & \frac{d P_{z}}{d t}=-\frac{\partial H}{\partial Z}, \\
\frac{d \Phi}{d t}=\frac{\partial H}{\partial P_{\varphi}}, & \frac{d P_{\varphi}}{d t}=-\frac{\partial H}{\partial \Phi},
\end{array}
$$

where $H=H\left(Z, \Phi, J_{x}, P_{z}, P_{\varphi}, t ; \epsilon\right)$ and $\left(Z, \Phi, J_{x}, P_{z}, P_{\varphi}\right)$ are Hamiltonian function and canonical variables, respectively, after some sufficient number of canonical transformations.

This reduced system describes the slow motion of the guiding center in a time-dependent magnetic and electric fields in a toroidal system. Up to the first order of $\epsilon$ the particle orbits are determined by Eq. (21) with the gyrophase $\vartheta_{x}=\int{ }^{t} \omega\left(t^{\prime}\right) d t^{\prime}+\vartheta_{x 0}$, where

$$
\omega(t) \equiv \omega\left(Z(t), \Phi(t), J_{x}, P_{z}(t), P_{\varphi}(t), t\right)=\frac{\partial H}{\partial J_{x}} .
$$

As mentioned above, the four-dimensional Hamiltonian guiding center equations have been derived and extensively studied previously in Refs. 6, 8, 7, 9-11, 13, and 14. These methods are based on the assumption of the existence of an adiabatic invariant, the magnetic moment $\mu$, and they use a magnetic coordinate system. However, it imposes a limitation on the form of the magnetic field in order to have canonical guiding center equations. It assumes a special form of the magnetic field to describe the case with chaotic field lines. ${ }^{8}$ In a more general approach given in Refs. 13 and 14 one may find a coordinate system in which one of the covariant components of the magnetic field $\mathbf{B}$ and the vector potential $\mathbf{A}$ vanish. However, in practice, it may be difficult to find such a coordinate system in a case with chaotic field lines. 
Our straightforward method allows one to obtain Hamiltonian guiding center equations for particles in timedependent magnetic and electric fields using a cylindrical coordinate system rather than a complex magnetic coordinate system. It makes these equations much simpler as compared to corresponding guiding center equations in a magnetic coordinate system. More important is that the use of the action variable $I_{x}$ as an adiabatic invariant makes more sense than using the magnetic moment $\mu$, because the action $I_{x}$ is related to the invariant $J_{x}$ of the Hamiltonian system, whereas the magnetic moment $\mu=m v_{\perp}^{2} / 2 B$ is related to the magnetic field only. Therefore, for instance, in the presence of the electric field the magnetic moment may be conserved with less accuracy than without. As shown in Ref. 20 one needs to modify the the magnetic moment $\mu$ in a strong sheared electric field in order to make it an adiabatic invariant. In our approach the action variable $I_{x}$ may be considered as an adiabatic invariant no matter whether the electric field is present or not. From the very beginning it is introduced for the whole system, which may include not only the effect of the magnetic field, but also the electric field within the plasma as well.

\section{SIMPLIFIED GUIDING CENTER EQUATIONS}

The equations of motion may significantly be simplified using the smallness of the poloidal magnetic field $B_{Z}$ in comparison with the toroidal field $B_{0}$ and the normalized velocity of particles $\left(u_{\varphi}, u_{z}\right)=\left(v_{\varphi}, v_{z}\right) / \omega_{c} R_{0}$ for the moderate energy particles. For the TEXTOR configuration $\left(R_{0}=175 \mathrm{~cm}, B_{0}\right.$ $=2 \mathrm{~T})$ the normalized velocity of the ion $H^{+}$is $u_{\text {ion }} \approx 2.9$ $\times 10^{-6} E_{i}^{1 / 2}$ and for electrons is $u_{\mathrm{e}} \approx 6.8 \times 10^{-7} E_{e}^{1 / 2}$, where $[E]=\mathrm{eV}$. One can see that for the particles up to the energy $E \leqslant 1 \mathrm{MeV}$ the normalized velocity $u$ is small. On the other hand, the normalized poloidal field $b_{z}=B_{Z} / B_{0}$ at the plasma edge is of order of $b_{z}=(\partial f / \partial x) /(1+x) \approx x / q \sim 10^{-1}$, where $q$ is a safety factor. Therefore, in the equations for the frequency $\omega_{x}(8)$ and the guiding center coordinate $x_{c}(9)$ one can neglect the terms proportional to $u^{2} \ll 1,\left|b_{z} u\right| \ll 1, b_{z}^{2}$ $\ll 1$. Thus we have the following simple equation for the guiding center $x_{c}$, i.e.,

$$
p_{z}+\ln (1+x)-(1+x) E_{x}=0,
$$

and the gyrofrequency $\omega_{x}$ is determined only by the toroidal magnetic field $B_{\varphi}=1 /(1+x)$ and the electric field $E_{x}$ $=-\partial \phi / \partial x$ :

$$
\omega_{0} \approx \frac{1}{1+x_{c}}\left[1-\left(1+x_{c}\right) E_{x}-\left(1+x_{c}\right)^{2} \frac{\partial E_{x}}{\partial x}\right]^{1 / 2} .
$$

Then the Hamiltonian (23) for the guiding center can be written as

$$
\begin{aligned}
H_{0}\left(I_{x}, Z, P_{z}, \Phi, P_{\varphi}\right)= & \omega_{0}\left(Z, \Phi, P_{z}, t\right) I_{x}+\frac{\left(P_{\varphi}-f\right)^{2}}{2\left(1+x_{c}\right)^{2}} \\
& +\phi\left(x_{c}, Z, \Phi, t\right),
\end{aligned}
$$

where $f=f\left(x_{c}, Z, \Phi, t\right), x_{c}=x_{c}\left(Z, \Phi, P_{z}, t\right)$. The equations of motion corresponding to Hamiltonian (30) are

$$
\begin{aligned}
& \frac{d Z}{d t}=\frac{\partial \omega_{0}}{\partial P_{z}} I_{x}+\frac{\partial U}{\partial x_{c}} \frac{\partial x_{c}}{\partial P_{z}}, \\
& \frac{d P_{z}}{d t}=-\frac{\partial \omega_{0}}{\partial Z} I_{x}+\frac{\left(P_{\varphi}-f\right)}{\left(1+x_{c}\right)^{2}} \frac{\partial f}{\partial Z}-\frac{\partial \phi}{\partial Z}-\frac{\partial U}{\partial x_{c}} \frac{\partial x_{c}}{\partial Z}, \\
& \frac{d \Phi}{d t}=\frac{\left(P_{\varphi}-f\right)}{\left(1+x_{c}\right)^{2}}, \\
& \frac{d P_{\varphi}}{d t}=-\frac{\partial \omega_{0}}{\partial \Phi} I_{x}+\frac{\left(P_{\varphi}-f\right)}{\left(1+x_{c}\right)^{2}} \frac{\partial f}{\partial \Phi}-\frac{\partial \phi}{\partial \Phi}-\frac{\partial U}{\partial x_{c}} \frac{\partial x_{c}}{\partial \Phi},
\end{aligned}
$$

where

$$
\frac{\partial U}{\partial x_{c}}=-\frac{\left(P_{\varphi}-f\right)^{2}}{\left(1+x_{c}\right)^{3}}-\frac{\left(P_{\varphi}-f\right)}{\left(1+x_{c}\right)^{2}} \frac{\partial f}{\partial x_{c}}+\frac{\partial \phi}{\partial x_{c}} .
$$

The action variable $I_{x}$ in these equations is considered as a constant of motion.

In the absence of the electric field $\left(E_{x} \equiv 0\right)$ one can obtain simple solutions of Eqs. (28) and (29),

$$
\omega_{x}=\frac{1}{1+x_{c}}=e^{p_{z}}, \quad x_{c}=e^{-p_{z}-1} .
$$

Then the relationships between the particle coordinates and its guiding center coordinates take the form

$$
\begin{aligned}
& x=x_{c}+\rho_{x} \sin \vartheta_{x}, \\
& z=Z+\rho_{x} \cos \vartheta_{x}, \quad \varphi=\Phi .
\end{aligned}
$$

The system of equations (31) and (32) is thereby further simplified because the frequency $\omega_{x}$ and the guiding center $x_{c}$ depend only on $P_{z}$.

\section{VERIFICATION OF GUIDING CENTER EQUATIONS}

In this section we verify the equations introduced for the guiding center of particles in a toroidal magnetic field in the absence and in the presence of external magnetic perturbations.

\section{A. Models of the equilibrium field and the magnetic perturbations}

Consider the standard magnetic field model in a large aspect ratio tokamak with circular cross section

$$
\mathbf{B}(r, \theta)=\frac{B_{0}}{1+r / R_{0} \cos \theta}\left(\mathbf{e}_{\varphi}+\frac{r}{q(r) R_{0}} \mathbf{e}_{\theta}\right),
$$

where $q(r)$ is a safety factor and $(r, \theta, \varphi)$ are toroidal coordinates, $\varepsilon=r / R_{0}(\epsilon \ll 1)$ is the inverse aspect ratio. In a coordinate system $(R, \varphi, Z)$ (or in normalized coordinates $x, \varphi, z)$ the equilibrium field (35) may be written via the vector potential $\mathbf{A}=\left(0, A_{\varphi}(x, z), A_{z}(R)\right)(\mathbf{B}=\nabla \times \mathbf{A})$ with the following components:

$$
\begin{aligned}
& A_{\varphi}(x, z)=\frac{B_{0} R_{0}^{2}}{R} \int \frac{d \psi}{q(r(\psi))}, \\
& A_{z}(R)=-B_{0} R_{0} \ln R,
\end{aligned}
$$

where $\psi=r^{2} / 2 R_{0}^{2}, r=R_{0} \sqrt{x^{2}+z^{2}}$. The safety factor is approximated by $q(r)=q_{0}+\left(q_{a}-q_{0}\right) r^{2} / a^{2}$. For the numerical 
calculations we choose the following TEXTOR parameters: the major radius $R_{0}=175 \mathrm{~cm}$, the minor radius $a=46 \mathrm{~cm}$, $q_{0}=0.75, q_{a}=3.5$.

We consider the external magnetic perturbations $\mathbf{B}_{1}$ created by the ideal DED coil configuration for the TEXTOR. ${ }^{17}$ The 16 identical helical coils are located on the inboard circumference of radius $r_{c}$ with the poloidal extention $2 \theta_{c}$. The asymptotic expressions for the perturbation field are found in Ref. 18. Using those formulas and (36) the normalized $\varphi$ component of the full vector potential A may be written as

$$
\begin{aligned}
f(x, z, \varphi, t)=\frac{R A_{\varphi}}{B_{0} R_{0}^{2}}= & \int \frac{d \psi}{q(r(\psi))} \\
& -\sum_{m} f_{m}(r) \cos (m \theta-n \varphi+\Omega t),
\end{aligned}
$$

where $m, n$ are poloidal and toroidal mode numbers, respectively, $f_{m}(r)$ is the radial-dependent amplitude of the $m$ mode:

$$
\begin{aligned}
& f_{m}(r)=\frac{B_{c} g_{m} r_{c}}{m B_{0} R_{0}} \sqrt{\frac{R}{R_{0}}}\left(\frac{r}{r_{c}}\right)^{m}, \\
& g_{m}=(-1)^{m+m_{0}} \frac{\sin \left(m-m_{0}\right) \theta_{c}}{\pi\left(m-m_{0}\right)}
\end{aligned}
$$

with the characteristic strength of the field $B_{c}$ $\approx \mu_{o} I_{d} n / \theta_{c} r_{c}$. The function $g_{m}$ describes the localized poloidal spectrum of the perturbation with the central mode $m_{0}\left(m_{0}=20\right)$. The toroidal mode number $n=4$ whereby the divertor current $I_{d}$ may reach up to $15 \mathrm{kA}$. It is also foreseen that the perturbed field can rotate with the frequency $\Omega$. The latter is one of the specific features of the DED.

For the numerical calculations we choose the following TEXTOR parameters: the major radius $R_{0}=175 \mathrm{~cm}$, the minor radius $a=46 \mathrm{~cm}, q_{0}=0.75, q_{a}=3.5$. The divertor current $I_{d}=15 \mathrm{kA}$, coil radius $r_{c}=53.25 \mathrm{~cm}$, and $\theta_{c}=\pi / 5$.

We have also studied the influence of the equilibrium potential of the electric field on particle orbits. The following model for the this field has been used $\phi(r)=-\phi_{0}[1$ $\left.-(r / a)^{4}\right]\left(\phi_{0}=e \Phi_{0} / m \omega_{c}^{2} R_{0}^{2}\right)$.

In Fig. 2 the poloidal dependence of the radial component $B_{r}$ of the perturbing magnetic field is shown at the different radial positions. One can see that the perturbation field is localized on the high field side. Such a perturbation creates the ergodic zone of chaotic field lines throughout the plasma edge. A Poincaré section of field lines at the edge region is shown in Fig. 3. A more detailed study of the field line ergodization under influence of the external magnetic perturbations in TEXTOR can be found in Ref. 18.

\section{B. The action variable $I_{x}$ and the magnetic momentum}

Below we study the behavior of the action variable $I_{x}$ and compare it with the magnetic moment $\mu$ for the motion of a hydrogen ion $\left(\mathrm{H}^{+}\right)$in the tokamak magnetic configuration described above. For TEXTOR tokamak parameters the time dependencies of the quantities $I_{x}$ and $\mu$ were computed by direct numerical integration of the six-dimensional

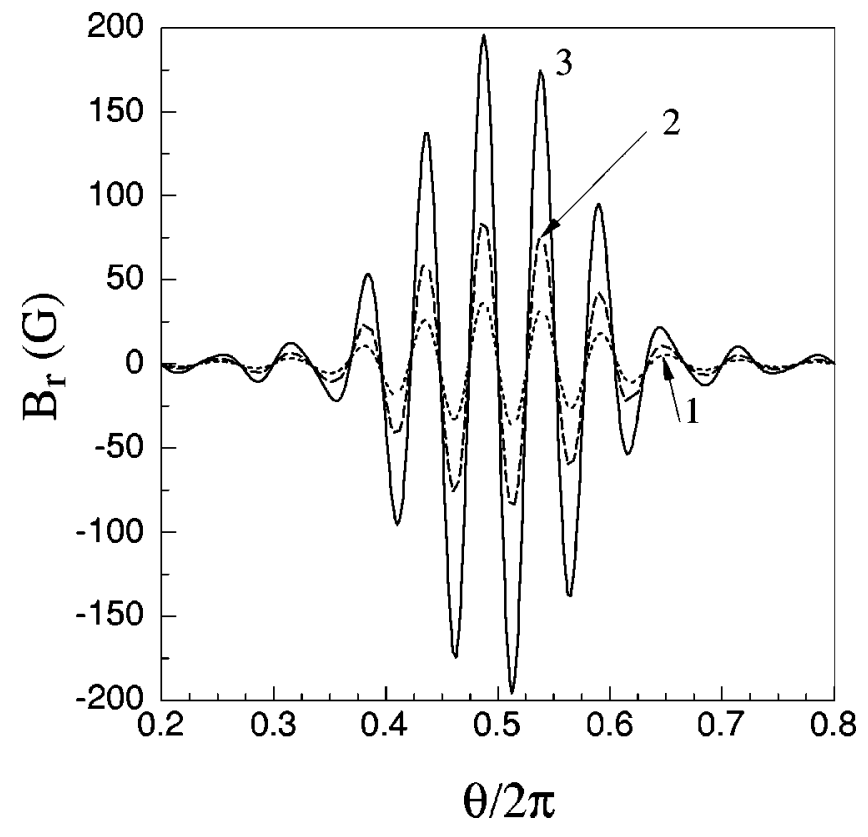

FIG. 2. Magnetic field perturbations $B_{r}$ as function of the poloidal angle $\theta$ at different radial distances: $r=42 \mathrm{~cm}$ (curve 1), $44 \mathrm{~cm}$ (curve 2), $46 \mathrm{~cm}$ (curve 3). Divertor current $I_{d}=15 \mathrm{kA}$, the coil radius $r_{c}=53.25 \mathrm{~cm}$, the poloidal extention $2 \theta_{c}=2 \pi / 5$.

Hamiltonian equations (1). The integration is carried out by the fifth-order Kunga-Kutta method with the accuracy $10^{-7}$. The action $I_{x}$ has been computed integrating (12) along the one closed circle on the $\left(x, p_{x}\right)$ plane. These calculations show that the action $I_{x}$ behaves similar to the magnetic moment $\mu$. Figures 4(a)-4(c) show the time dependencies of the $I_{x}$ and $\mu(t)$ normalized to the initial value of the magnetic moment $\mu(0)$ in the absence (a), in the presences of the external static (b) and time-dependent (c) magnetic perturbations with the frequency $f=\Omega / 2 \pi=10 \mathrm{kHz}$ (37), respectively. The curve 1 describes the normalized magnetic moment $\mu(t) / \mu(0)$, and the curve 2 , the normalized action $I_{x}(t) / I_{x}(0)$. The initial coordinate of the hydrogen ion $\left(\mathrm{H}^{+}\right)$ was taken at $r=43 \mathrm{~cm}, \theta_{0}=0$, and the energy $E=1 \mathrm{keV}$ the relative perpendicular energy $\lambda=\mu B_{0} / E=0.5$. The perturbed magnetic field at this distance is sufficiently strong to

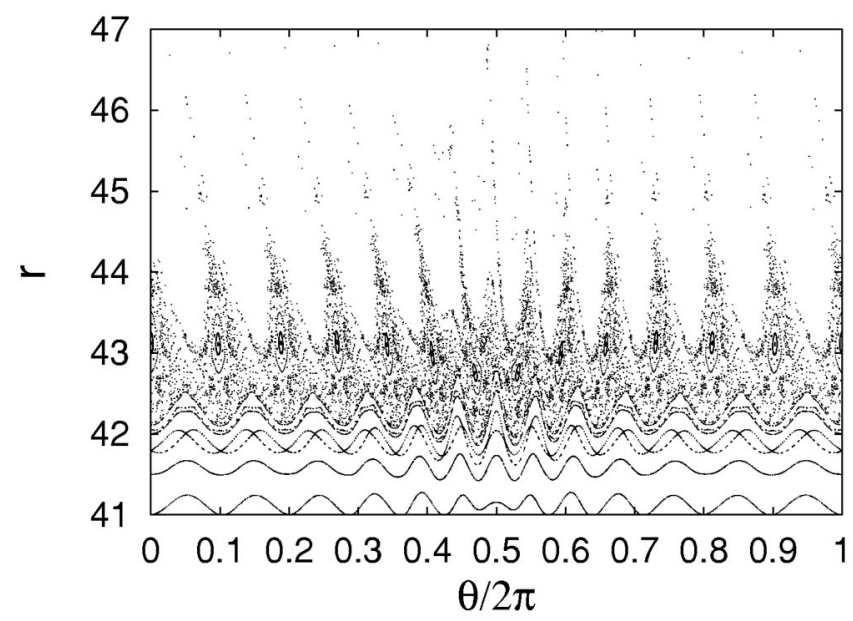

FIG. 3. Poincaré section of magnetic field lines on the $(\theta, r)$ plane at the plasma edge. Parameters are the same as in Fig. 2. 
(a)

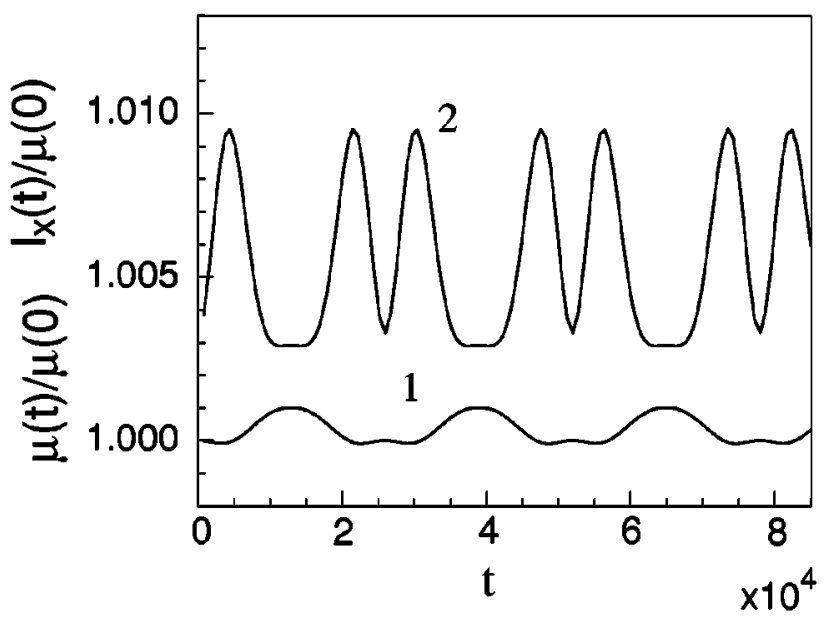

(b)

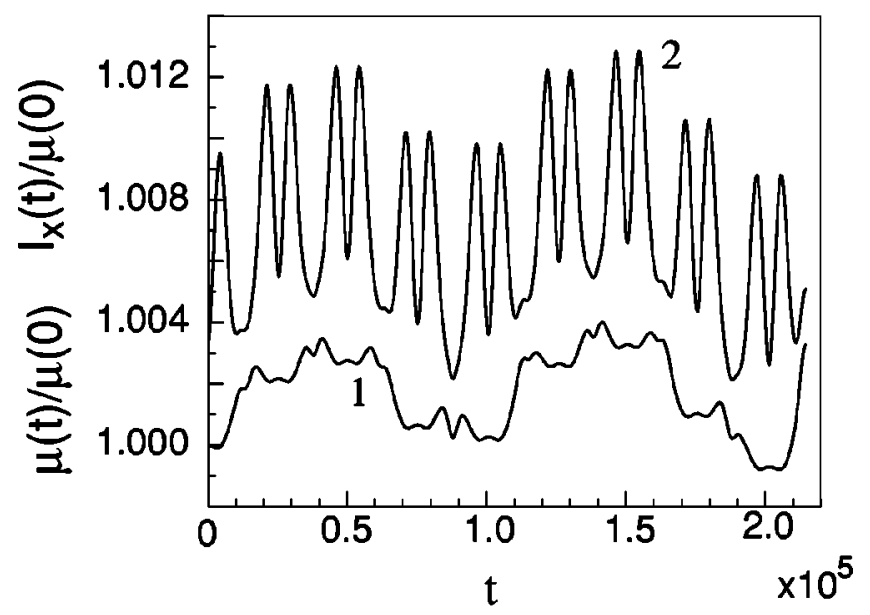

(c)

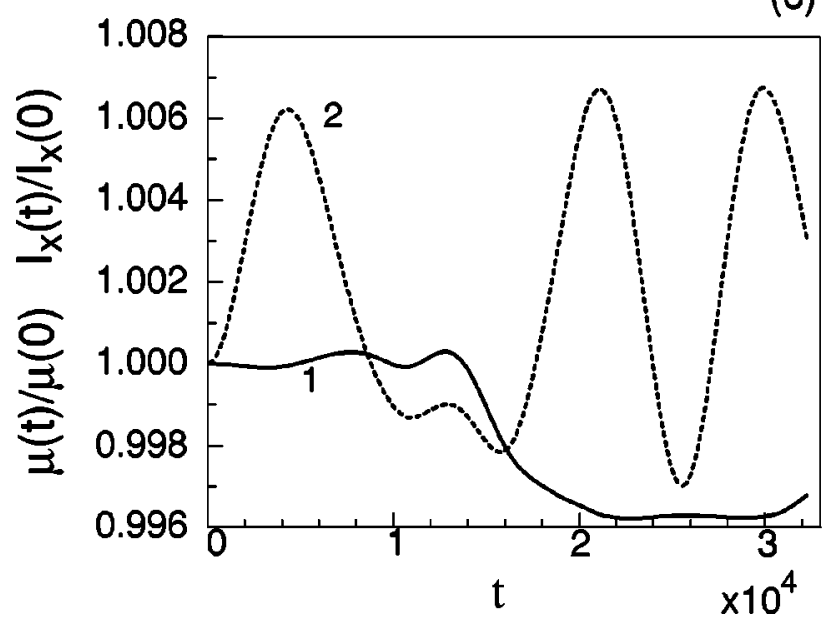

FIG. 4. Time dependencies of the normalized magnetic moment $\mu(t) / \mu(0)$ (curve 1) and the normalized action variable $I_{x}(t) / \mu(0)$ [or $I_{x}(t) / I_{x}(0)$ ] (curve 2) of a hydrogen ion $\left(\mathrm{H}^{+}\right)$with energy $E=1 \mathrm{keV}$ and $\lambda=0.5$ : (a) in the absence of magnetic perturbations; (b) in the presence of the static perturbations; (c) in the presence of time-dependent perturbations with $\Omega$ $=10^{4} \mathrm{~Hz}$.

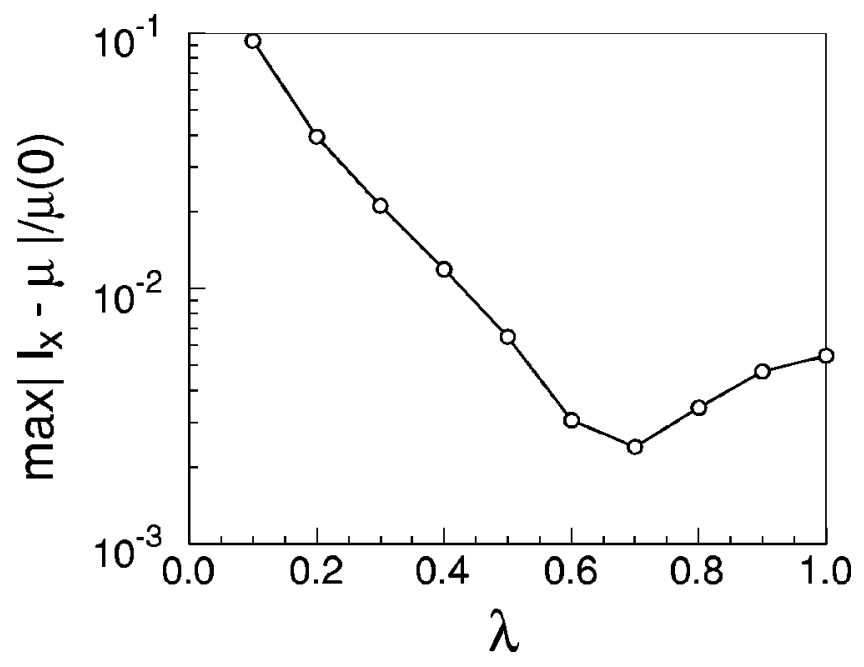

FIG. 5. Maximum deviation of the action $I_{x}(t)$ from the magnetic moment $\mu(t)$ normalized to $\mu(0), \max \left|I_{x}(t)-\mu(t)\right| / \mu(0)$ versus the relative perpendicular energy $\lambda$ in the absence of external perturbations. Ion energy $\left(\mathrm{H}^{+}\right)$ $E=50 \mathrm{eV}$.

produce chaotic field lines. In the last case shown in Fig. 4(c) the particle leaves the plasma region after hitting the divertor plate located at $r_{d}=47.7 \mathrm{~cm}$. From calculations it follows that (i) the deviation of the action $I_{x}(t)$ from the magnetic moment $\mu(t)$ is sufficiently small during large time intervals for both cases, in the absence and presence of magnetic perturbations; (ii) although the action $I_{x}(t)$ varies in time with larger amplitude than those of the magnetic moment $\mu(t)$ whereby its relative deviations from the initial value $I_{x}(0)$ remain small. The amplitude of oscillation of $I_{x}(t)$ and its deviation from the magnetic moment depends on the relative perpendicular energy according $\lambda=\mu B_{0} / E$. The dependence of the maximal deviations of the action $I_{x}(t)$ from $\mu(t)$ normalized to $\mu(0), \max \left|I_{x}(t)-\mu(t)\right| / \mu(0)$ on $\lambda$ are shown in Fig. 5 for the hydrogen ion of the energy $50 \mathrm{eV}$ and in the absence of magnetic perturbations. One can see that the difference becomes larger for the small values of $\lambda$, where values of $I_{x}(t)$ and $\mu(t)$ are themselves small.

The cases of high energetic particles and the influence of the electric field have been studied as well. In Figs. 6(a) and 6(b) the time dependencies of $I_{x}(t)$ and $\mu(t)$ are shown for the particle of energy $E=1 \mathrm{MeV}$ without electric field, and for $E=1 \mathrm{keV}$ with the electric field of the potential amplitude $e \Phi_{0}=400 \mathrm{eV}$. The full curves 1 correspond to $I_{x}(t) / I_{x}(0)$, and the dotted curves 2 describe $\mu(t) / \mu(0)$. One can see that in these cases the amplitude of variation of the action $I_{x}(t)$ is even several times smaller than the one of the magnetic moment $\mu(t)$. Therefore, similar to the magnetic moment $\mu$, the action variable $I_{x}$ may be considered as an adiabatic invariant.

According to (12) the adiabatic invariant $I_{x}$ is the ratio of the radial energy $E_{r}$ to the $\omega_{x}: I_{x}=E_{r} / \omega_{x}$, similar to the definition of the magnetic moment $\mu=E_{\perp} / \omega_{c}$ (in normalized variables). The adiabatic invariant $I_{x}$, according to the definition of $\omega_{x}(8)$, includes the effect of electric field:

$$
I_{x}=I_{x 0}\left[1+\left(1+x_{c}\right)^{2} \frac{\partial^{2} \phi}{\partial x^{2}}\right]^{-1 / 2} \approx I_{x 0}\left[1+\frac{e}{2 m \omega_{c}^{2}} \frac{\partial E_{R}}{\partial R}\right],
$$


(a)

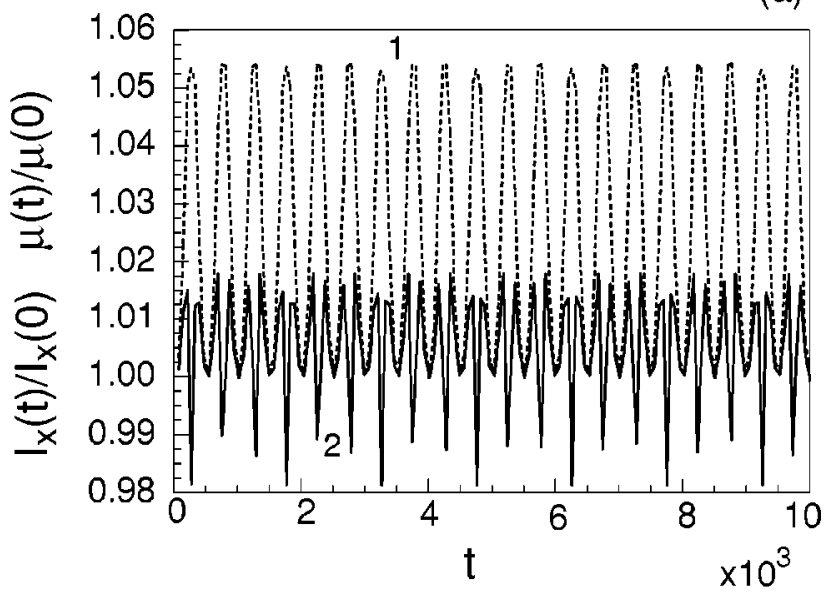

(b)

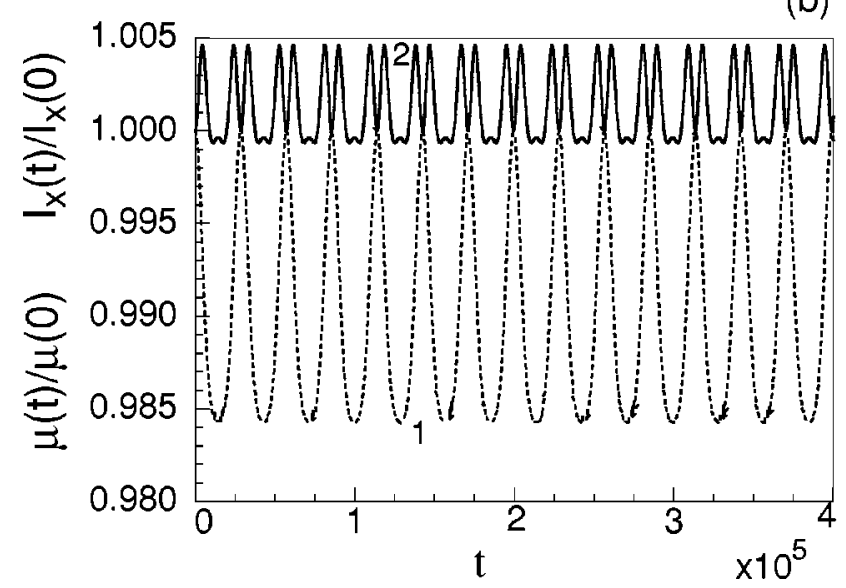

FIG. 6. The same as Fig. 4 but the energetic particle of energy $E=1 \mathrm{MeV}$ (a) and in the presence of electric field (b). Parameter $\lambda=0.5$.

where $E_{R}=-\partial \Phi / \partial R$ is a radial component of the electric field, $I_{x 0}$ is an adiabatic invariant in the absence of the electric field. This formula is similar to the one for the magnetic moment $\mu$ in a strongly sheared electric field obtained in Ref. 20.

\section{Particle orbits}

The guiding center orbits are obtained using the simplified guiding center equations (31) and (32) supposing that the action variable $I_{x}$ is a constant of motion. This well describes the particle's orbits in toroidal geometry, thereby reproducing the well-known characteristics of guiding center motion, like the banana orbits on the poloidal $(x, z)$ plane and toroidal precession motion on the $(R, \varphi)$ plane. First using the Hamiltonian (30) we estimate the shift of the drift orbits from the magnetic surfaces and the width of the banana orbits.

Consider the magnetic surface $f(x, z)=P_{\varphi}^{(M)}=$ const. Suppose that the guiding center of the particle crosses the point $\left(x=x_{m}>0, z=0\right)$ at the low field side. The magnetic and the drift surfaces crossing this point are given by formulas

$f(x, z)=P_{\varphi}^{(M)}=P_{\varphi}-\sigma\left(1+x_{m}\right) \sqrt{2\left(H-\omega_{x}\left(x_{m}\right) I_{x}\right)}$, $f(x, z)=P_{\varphi}-\sigma(1+x) \sqrt{2\left(H-\omega_{x}(x) I_{x}\right)}$,

respectively. Here $\sigma=v_{\varphi} /\left|v_{\varphi}\right|$ determines the direction of particle motion: $\sigma=1$ if a particle moves the along positive direction of the toroidal angle $\varphi$, and $\sigma=-1$ if it moves in the opposite direction. In the absence of the electric field we have $\omega_{x}(x)=1 /(1+x)$. Let $r(\theta)$ and $r_{M}(\theta)$ be radial coordinates as a function of the poloidal angle $\theta$ of the drift and the magnetic surfaces, respectively. According to (39) and (40) the relation between these coordinates is given

$$
\begin{aligned}
f(r \cos \theta, r \sin \theta)-f\left(r_{M} \cos \theta, r_{M} \sin \theta\right) & =\sigma_{i}\left(1+\frac{r_{M}(0)}{R_{0}}\right) \sqrt{2\left(H-\frac{I_{x}}{1+r_{M}(0) / R_{0}}\right)} \\
& -\sigma_{f}\left(1+\frac{r \cos \theta}{R_{0}}\right) \sqrt{2\left(H-\frac{I_{x}}{1+r \cos \theta / R_{0}}\right)} .
\end{aligned}
$$

In (39) $\sigma_{f}=\sigma_{i}$ for the passing particles. For the trapped particles with banana orbits $\sigma_{f}=\sigma_{i}$ at the branch of the orbit which has a common point with a magnetic surface, and $\sigma_{f}=-\sigma_{i}$ at the other branch.

For the passing particles $H>I_{x} /\left(1-r(\pi) / R_{0}\right)$ and for the trapped particles $H<I_{x} /\left(1-r(\pi) / R_{0}\right)$. The turning poloidal angle $\theta_{c}$ of the banana orbits of the trapped particles is determined as $r\left(\theta_{c}\right) \cos \theta_{c} / R_{0}=\lambda_{I}-1$, where $\lambda_{I}=I_{x} / H$.

Consider the case of small deviations of the drift surface from the magnetic one. Then using the relations $\partial f / \partial x$ $=x / q, \partial f / \partial z=z / q$, one can show that

$$
\begin{aligned}
r-r_{M} \approx & \sqrt{2 H} \frac{q(r) R_{0}^{2}}{r}\left[\sigma_{i}\left(1+\frac{r(0)}{R_{0}}\right) \sqrt{1-\frac{\lambda_{I}}{1+r(0) / R_{0}}}\right. \\
& \left.-\sigma_{f}\left(1+\frac{r \cos \theta}{R_{0}}\right) \sqrt{1-\frac{\lambda_{I}}{1+r \cos \theta / R_{0}}}\right] .
\end{aligned}
$$

The maximum deviation occurs for the weakly trapped particles, $\sigma_{f}=-\sigma_{i}, \lambda_{I}=1-r(\pi) / R_{0}$, at the low field side $\theta$ $=0$ :

$$
\begin{aligned}
\Delta_{t}=\left|r-r_{M}\right| & =4 E^{1 / 2} q R_{0}\left(\frac{R_{0}+r}{r}\right)^{1 / 2} \\
& =4 \frac{q R_{0}}{\omega_{c} r} \sqrt{\frac{J_{r} r}{m R_{0}}}\left(\frac{R_{0}+r}{R_{0}-r}\right)^{1 / 2},
\end{aligned}
$$

where $J_{x}=m \omega_{c}^{2} R_{0}^{2} I_{x}$ and it is supposed that $r=r(0)$ $\approx r(\pi)$. For the just passing particles $\left(\sigma_{f}=\sigma_{i}, \lambda_{I}=1\right.$ $-r / R_{0}$ ) the maximum deviation occurs on the high field side $\theta=\pi: \Delta_{p}=\Delta_{t} / 2$.

For large aspect ratio $R_{0} / r \gg 1$ the formula (43) coincides with the one obtained by Berk and Galeev, ${ }^{29}$ if the action variable $J_{x}$ is replaced by $m \mu B_{0}: \Delta_{t}$ $=4\left(\mu B_{0} r / R\right)^{1 / 2} / \omega_{c} \theta$, where $\theta=\iota r /\left.R\right|_{r=r_{M}}, \iota=1 / q$.

We have estimated $\Delta_{t}$ for the TEXTOR parameters. For the weakly trapped particles of energy $E=1 \mathrm{keV}$ with $I_{x} / E$ $=0.749$ and the just passing particles of the same energy $\lambda$ $=I_{x} / E=0.748$. From the numerical integration of Hamiltonian equations we have obtained $\Delta_{t}=4.191 \mathrm{~cm}$, for passing particle $\Delta_{p}=1.975 \mathrm{~cm}$ which are sufficiently close the estimations (43): $\Delta_{t}=3.972 \mathrm{~cm}$ and $\Delta_{p}=1.986 \mathrm{~cm}$, respec- 
(a)

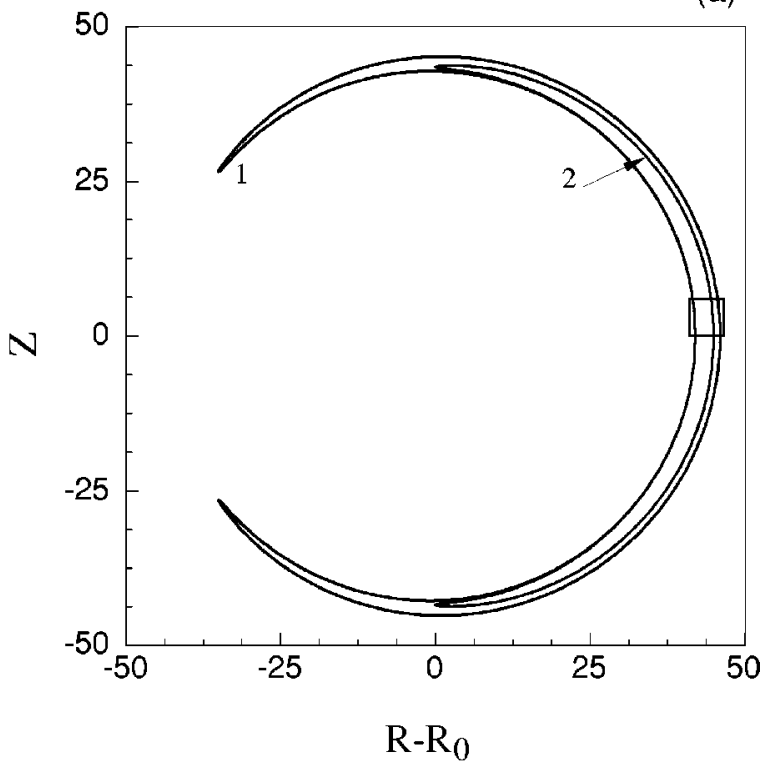

(b)

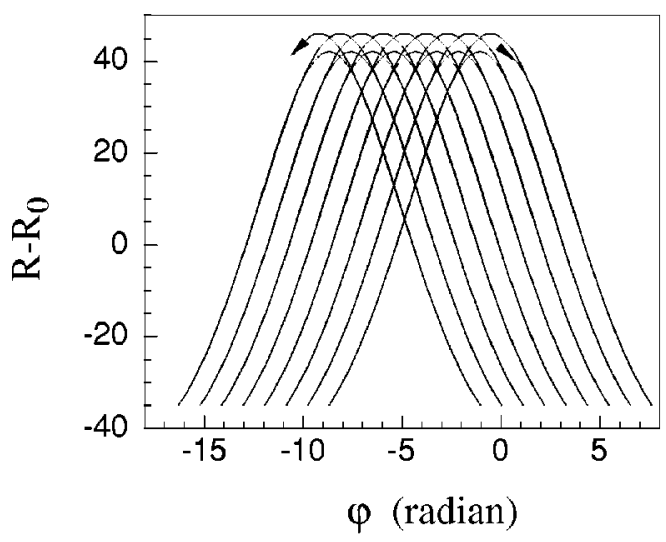

(c)

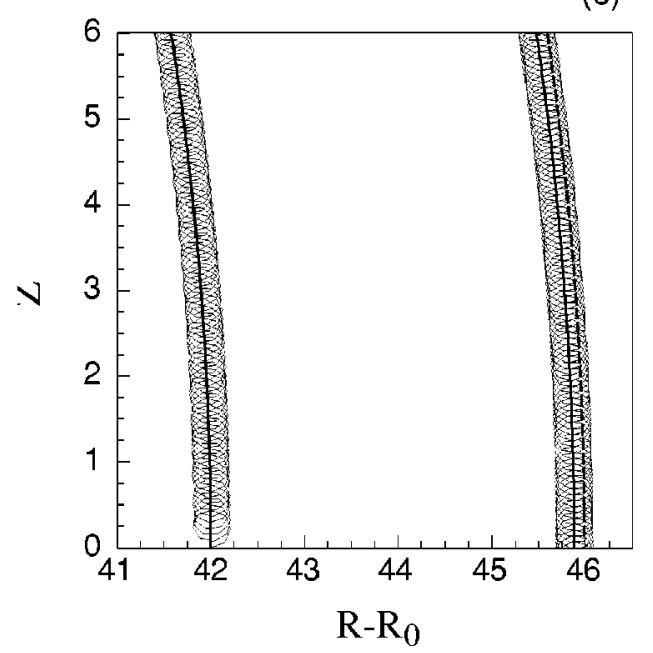

FIG. 7. (a) Guiding center orbits of trapped ions $\left(\mathrm{H}^{+}\right)$in the poloidal plane $\left(R-R_{0}, Z\right): \lambda=0.8$ (curve 1), 1 (curve 2); (b) toroidal procession of the guiding center orbit of a trapped ion on the $\left(\varphi, R-R_{0}\right)$ plane. (c) Expanded view of the rectangular region shown in (a) of the gyrating orbit and its guiding center with (full curves) and without (dotted curve) electric field $\left(e \Phi_{0}=100 \mathrm{eV}, \lambda=0.8\right)$. Energy of the ion $E=1 \mathrm{keV}$.

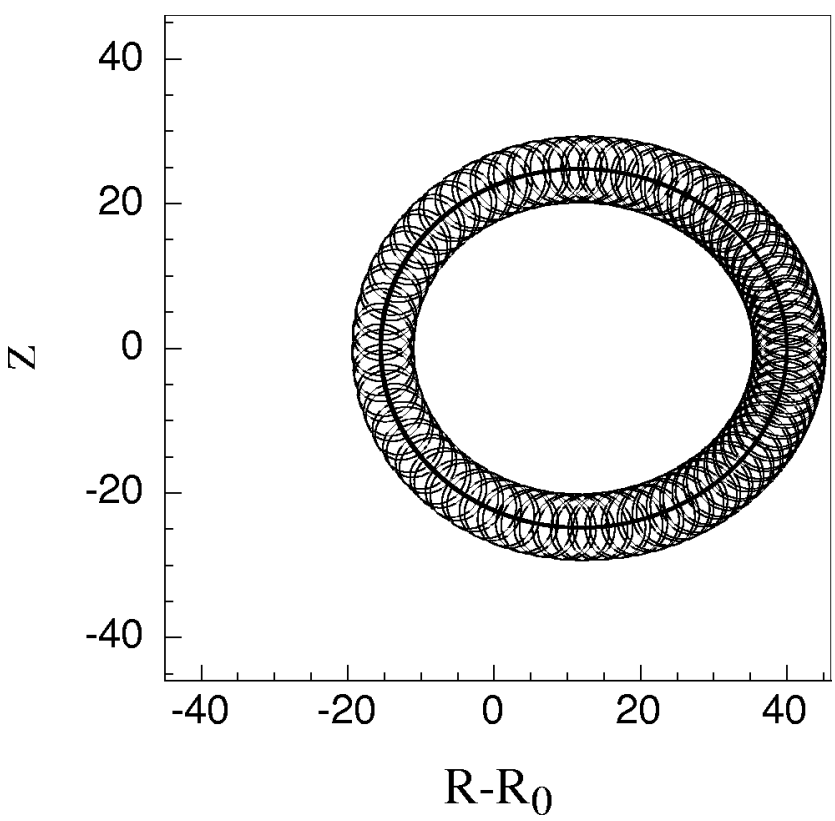

FIG. 8. Gyrating orbit of the counter-passing $\left(\sigma=v_{\varphi} /\left|v_{\varphi}\right|=-1\right)$ energetic ion $\left(\mathrm{H}^{+}\right)$and its guiding center on the poloidal plane $\left(R-R_{0}, Z\right)$. The guiding center orbit is obtained using the simplified Hamiltonian equations. Energy $E=1 \mathrm{MeV}$ and $\lambda=0.5$.

tively. For the particles of energy $E=100 \mathrm{eV}$ we have $\Delta_{t}$ $=1.276 \mathrm{~cm}, \Delta_{p}=0.618 \mathrm{~cm}$ from the numerical integration and $\Delta_{t}=1.254 \mathrm{~cm}, \Delta_{p}=0.627 \mathrm{~cm}$ according to (43). The estimates of Berk and Galeev give smaller values by factor $\sqrt{\left(R_{0}-r\right) /\left(R_{0}+r\right)}=1.277$.

Below we present examples of some orbits obtained using the simplified Hamiltonian guiding center equations and compare them with those obtained by direct integration $6 \mathrm{D}$ Hamiltonian system (1). In Fig. 7 we have shown the trapped hydrogen ion $\left(\mathrm{H}^{+}\right)$of energy $E=1 \mathrm{eV}$ with $\lambda=0.8$ and 1.0 with and without electric field: (a) describes the trapped banana orbits on the $\left(R-R_{0}, Z\right)$ plane without electric field (curve 1 corresponds to $\lambda=0.8$, and curve $2-\lambda=1$ ); (b) shows the toroidal precession of the particle orbit on the $(\varphi, R)$ plane $(\lambda=0.8)$, (c) presents the expanded view of the rectangular region of the trapped orbit $(\lambda=0.8)$ drawn in (a) in which the gyrating particle orbit and its guiding center are shown. In the latter case the full curves describe the orbit in the presence of the electric field $\left(e \Phi_{0}=100 \mathrm{eV}\right)$, and the dotted curve corresponds to the orbits in its absence. The gyrating orbit is obtained by direct integration of the $6 \mathrm{D}$ Hamiltonian equations (1). Similar orbits of the gyrating high-energetic counter-passing particle $\left(\sigma=v_{\varphi} /\left|v_{\varphi}\right|=-1\right)$ of energy $E=1 \mathrm{MeV}(\lambda=0.5)$ and its guiding center are presented in Fig. 8. One can see from Figs. 7(c) and 8 that the simplified equations (31) and (32) well reproduce the guiding center orbits of gyrating particles. They also describe the shrinking of the trapped particle orbits in the presence of the electric field [see Fig. 7(c)].

Finally, consider the chaotic orbit of particle in the presence of the external magnetic perturbations (37). In Fig. 9 a chaotic particle orbit at the plasma edge obtained by the guiding center equations (31) and (32) (thick curve 1) and 
(a)

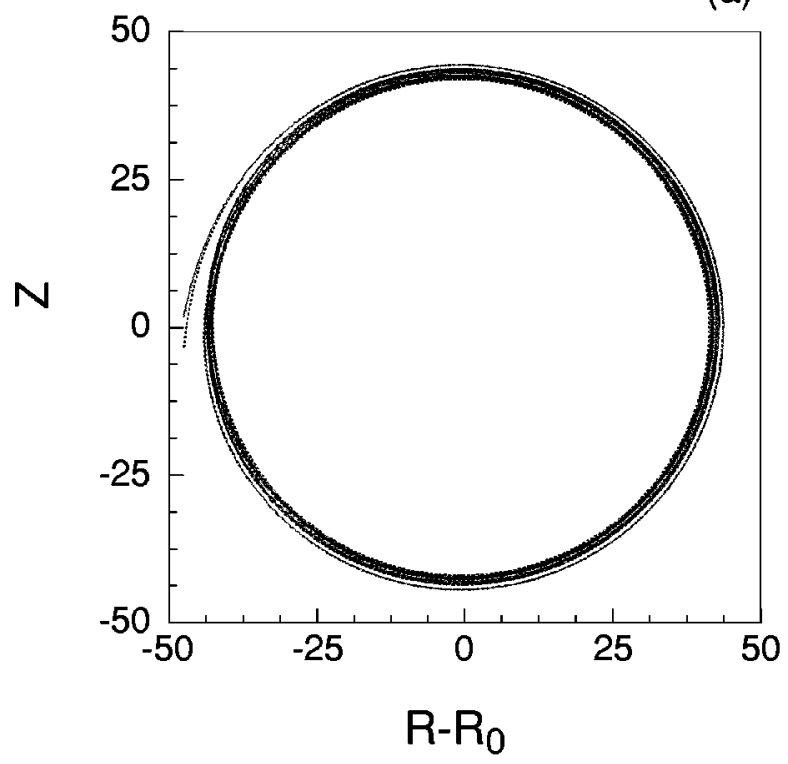

(b)

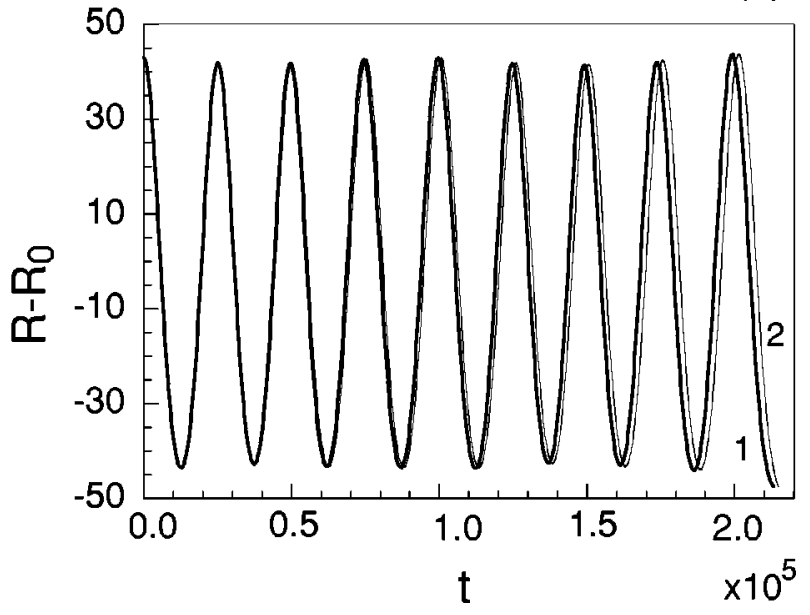

FIG. 9. (a) Chaotic orbit of a co-passing $\left(\sigma=v_{\varphi} /\left|v_{\varphi}\right|=1\right)$ ion $\left(\mathrm{H}^{+}\right)$on the poloidal plane $\left(R-R_{0}, Z\right)$. Thick curve 1 corresponds to the guiding center orbit obtained by the simplified Hamiltonian equations, thin one (curve 2), to the exact orbit. (b) Time dependence of the radial coordinate $R-R_{0}$ of the same orbits as in (a). Energy $E=1 \mathrm{keV}$ and $\lambda=0.5$.

the exact equations (1) (thin curve 2) are shown. The counter-passing $(\sigma=-1)$ particle of energy $E=1 \mathrm{keV}$ and $\lambda=0.5$ with initial coordinates $(r=43 \mathrm{~cm}, \theta=0)$ at $t=0$ hits the divertor plate at $r_{d}=47.7 \mathrm{~cm}$ at the time instant $t=2.26$ $\times 10^{5}$ : (a) shows the orbits on the poloidal section $[(R$ $-R_{0}, z$ ) plane], and (b) the time dependence of the radial coordinate $R(t)$. Although the comparison of the guiding center orbits with the gyrating orbits for the chaotic motion does not make much sense for long time intervals because of sensitivity of exponentially diverging orbits on small uncertainty of the initial coordinates ${ }^{11}$ nevertheless at the initial stage the guiding center orbit of the chaotic particle closely reproduces its gyrating orbit obtained by the exact $6 \mathrm{D}$ equations.

\section{CONCLUSIONS}

We have developed a new Hamiltonian theory of guiding center motion in magnetic and electric fields of tokamak magnetic confinement devices. The equations of motion for guiding center are obtained from the original 6D Hamiltonian equations in a cylindrical coordinate system for a charged particle by applying a special canonical transform of its fast oscillating variables to slowly varying guiding center variables. The transformed new Hamiltonian system describes the guiding center motion in $6 \mathrm{D}$ phase space for the slow changing variables. These equations may be reduced to a 4D system if the gyrofrequency of the radial oscillations is much larger than the macroscopic transit frequency $L / v_{t h}$ of the system. In this case there exists an adiabatic invariant of the motion, i.e., the action variable, related to the fast radial oscillations of particle.

These Hamiltonian guiding center equations have several advantages in comparison to previously proposed Hamiltonian guiding center equations formulated using the existence of the adiabatic invariant of motion, the magnetic moment of particles. Unlike those theories which use a rather complex magnetic coordinate system we have obtained the canonical guiding center equations of motion in a cylindrical coordinate system. These equations are much simpler than those in a magnetic coordinate system, and they allow one to treat the cases of nonaxisymmetric and time-dependent magnetic and electric perturbations without additional formal assumptions on their form. In the presented work we have restricted ourselves to the case of a simplified equilibrium toroidal magnetic field, $B_{\varphi}=B_{0} R_{0} / R$. This case may be useful to study the particle orbits in tokamaks with the destroyed magnetic surfaces, and when the high-precision analyzes of the experiment is not so important. The method may nonetheless be generalized to the cases of more realistic equilibrium magnetic fields.

The Hamiltonian form of equations is very useful from the computational point of view. First of all this gives a rather simple form as compared to those ones in magnetic coordinate systems. On the other hand, one can integrate them using the powerful methods of symplectic integration ${ }^{26}$ and, in particular, the methods of symplectic mappings. ${ }^{27}$

\section{ACKNOWLEDGMENTS}

The authors are grateful to Professor G. Eilenberger and Dr. G. Fuchs for very useful discussions and suggestions.

${ }^{1}$ C. S. Gardner, Phys. Rev. 115, 791 (1959).

${ }^{2}$ C. S. Gardner, Phys. Fluids 9, 1997 (1966).

${ }^{3}$ H. E. Mynick, Phys. Rev. Lett. 43, 1019 (1979).

${ }^{4}$ H. E. Mynick, Phys. Fluids 23, 1888 (1980); 23, 1897 (1980).

${ }^{5}$ H. Weitzner, Phys. Fluids 24, 2280 (1981).

${ }^{6}$ R. G. Littlejohn, Phys. Fluids 24, 1730 (1981).

${ }^{7}$ R. G. Littlejohn, J. Plasma Phys. 29, 111 (1983).

${ }^{8}$ R. B. White, A. H. Boozer, and R. Hay, Phys. Fluids 25, 575 (1982).

${ }^{9}$ A. H. Boozer, Phys. Fluids 27, 2441 (1984).

${ }^{10}$ R. B. White and M. S. Chance, Phys. Fluids 27, 2455 (1984).

${ }^{11}$ R. B. White, Phys. Fluids B 2, 845 (1990).

${ }^{12}$ A. H. Boozer and R. B. White, Phys. Rev. Lett. 49, 786 (1982).

${ }^{13}$ J. D. Meiss and R. D. Hazeltine, Phys. Fluids B 2, 2563 (1990).

${ }^{14}$ H. Wobig and D. Pfirsch, Plasma Phys. Controlled Fusion 43, 695 (2001).

${ }^{15}$ A. H. Boozer, Phys. Fluids 26, 1288 (1983).

${ }^{16} \mathrm{Ph}$. Ghendrih, A. Grossman, and H. Cape, Plasma Phys. Controlled Fusion 38, 1653 (1996).

${ }^{17}$ Special issue on Dynamic Ergodic Divertor, edited by K. H. Finken, Fusion Eng. Des. 37, 335 (1997). 
${ }^{18}$ S. S. Abdullaev, K. H. Finken, and K. H. Spatscheck, Phys. Plasmas 6, 153 (1999).

${ }^{19}$ J. Carlsson, Phys. Plasmas 8, 4725 (2001).

${ }^{20}$ M. Dirickx and B. Weyssow, J. Plasma Phys. 59, 211 (1998).

${ }^{21}$ H. Weitzner, Phys. Plasmas 2, 3595 (1995).

${ }^{22}$ H. Weitzner, Phys. Plasmas 6, 420 (1999).

${ }^{23}$ R. M. Kulsrud, Phys. Rev. 106, 205 (1957)

${ }^{24}$ F. Hertweck and A. Schlüter, Z. Naturforsch. A 12A, 844 (1957).

${ }^{25}$ R. Meyer, Z. Angew. Math. Phys. 24, 293 (1973).
${ }^{26}$ J. M. Sanz-Serna, "Symplectic integrators for Hamiltonian problems: An overview," Acta Numerica (Cambridge University Press, Cambridge, 1992), pp. 243-286.

${ }^{27}$ S. S. Abdullaev, J. Phys. A 32, 2745 (1999).

${ }^{28}$ V. I. Arnold, V. V. Kozlov, and A. I. Neishtadt, "Mathematical aspects of classical and celestial mechanics," Encyclopeadia of Mathematical Sciences. Dynamical Systems, Vol. III (Springer-Verlag, Berlin, 1988).

${ }^{29}$ H. L. Berk and A. A. Galeev, Phys. Fluids 10, 441 (1967). 